\title{
Secular trends in acute dialysis after elective major surgery - 1995 to 2009
}

\author{
Nausheen F. Siddiqui MD, Steven G. Coca DO MS, Philip J. Devereaux MD PhD, Arsh K. Jain MD, Lihua Li MSc, \\ Jin Luo MSc, Chirag R. Parikh MD PhD, Michael Paterson MSc, Heather Thiessen Philbrook MMath, \\ Ron Wald MDCM MPH, Michael Walsh MD MSc, Richard Whitlock MD MSc, Amit X. Garg MD PhD
}

\begin{abstract}
- ABSTRACT
Background: Acute kidney injury is a serious complication of elective major surgery. Acute dialysis is used to support life in the most severe cases. We examined whether rates and outcomes of acute dialysis after elective major surgery have changed over time.

Methods: We used data from Ontario's universal health care databases to study all consecutive patients who had elective major surgery at 118 hospitals between 1995 and 2009. Our primary outcomes were acute dialysis within 14 days of surgery, death within 90 days of surgery and chronic dialysis for patients who did not recover kidney function.

Results: A total of 552672 patients underwent elective major surgery during the study period,

2231 of whom received acute dialysis. The incidence of acute dialysis increased steadily from $0.2 \%$ in 1995 (95\% confidence interval [Cl] 0.15 $0.2)$ to $0.6 \%$ in $2009(95 \% \mathrm{Cl} 0.6-0.7)$. This increase was primarily in cardiac and vascular surgeries. Among patients who received acute dialysis, 937 died within 90 days of surgery (42.0\%, 95\% Cl 40.0-44.1), with no change in 90day survival over time. Among the 1294 patients who received acute dialysis and survived beyond 90 days, 352 required chronic dialysis $(27.2 \%$ 95\% Cl 24.8-29.7), with no change over time.

Interpretation: The use of acute dialysis after cardiac and vascular surgery has increased substantially since 1995 . Studies focusing on interventions to better prevent and treat perioperative acute kidney injury are needed.
\end{abstract}

$\mathrm{M}$ ore than 230 million elective major surgeries are done annually worldwide. ${ }^{1}$ Acute kidney injury is a serious complication of major surgery. It represents a sudden loss of kidney function that affects morbidity, mortality and health care costs. ${ }^{2}$ Dialysis is used for the most severe forms of acute kidney injury. In the nonsurgical setting, the incidence of acute dialysis has steadily increased over the last 15 years, and patients are now more likely to survive to discharge from hospital. ${ }^{3-5}$ Similarly, in the surgical setting, the incidence of acute dialysis appears to be increasing over time, ${ }^{6-10}$ with declining inhospital mortality., ${ }^{8,10,11}$

Although previous studies have improved our understanding of the epidemiology of acute dialysis in the surgical setting, several questions remain. Many previous studies were conducted at a single centre, thereby limiting their generalizability. ${ }^{6,12-14}$ Most multicentre studies were conducted in the nonsurgical setting and used diagnostic codes for acute kidney injury not requiring dialysis; however, these codes can be inaccurate..$^{15,16}$

In contrast, a procedure such as dialysis is eas- ily determined. The incidence of acute dialysis after elective surgery is of particular interest given the need for surgical consent, the severe nature of the event and the potential for mitigation. The need for chronic dialysis among patients who do not recover renal function after surgery has been poorly studied, yet this condition has a major affect on patient survival and quality of life. ${ }^{17}$ For these reasons, we studied secular trends in acute dialysis after elective major surgery, focusing on incidence, 90-day mortality and need for chronic dialysis.

\section{Methods}

\section{Study design and setting}

We used data from Ontario's linked provincial health care databases, held at the Institute for Clinical Evaluative Sciences, for all consecutive patients who had elective major surgery between Jan. 1, 1995, and Dec. 31, 2009. All encounters with the health care system in Ontario are recorded and anonymized using encrypted identifiers to track individual patients over time. We conducted the study according to a prespecified
Competing interests: None declared.

This article has been peer reviewed.

Correspondence to: Amit Garg, amit.garg@1hsc.on.ca

CMAJ 2012. DOI:10.1503 /cmaj.110895 
protocol. The study was approved by the Sunnybrook Health Sciences Centre Research Ethics Board in Toronto, Ont.

\section{Data sources}

We used three linked databases: the Canadian Institute for Health Information's discharge abstract database records all admissions to hospital and includes information on diagnoses and procedures performed; the Ontario Health Insurance Plan database contains information on all physician claims for inpatient and outpatient services; and the Registered Persons Database contains vital statistics on all permanent residents of Ontario. These databases have been used extensively to research health outcomes. ${ }^{18-22}$ Whenever possible, we defined patient characteristics, surgery characteristics and outcomes using database codes that have been proven reliable when compared with manual chart review (Appendices 1 and 2, available at www.cmaj.ca/lookup /suppl/doi:10.1503/cmaj.110895/-/DC1).

\section{Patients}

We considered patients 18 years of age and older who underwent elective surgery with an over-

Table 1: Characteristics of patients undergoing major elective surgery in Ontario, 1995-2009

\begin{tabular}{|c|c|c|c|c|c|}
\hline \multirow[b]{2}{*}{ Characteristic } & \multicolumn{4}{|c|}{ No. $(\%)$} & \multirow[b]{2}{*}{$p$ value* } \\
\hline & $\begin{array}{l}1995-1997 \\
3 \text { years }\end{array}$ & $\begin{array}{l}1998-2001 \\
4 \text { years }\end{array}$ & $\begin{array}{l}2002-2005 \\
4 \text { years }\end{array}$ & $\begin{array}{l}2006-2009 \\
4 \text { years }\end{array}$ & \\
\hline Type of surgeryt & $n=136453$ & $n=162113$ & $n=133268$ & $n=120838$ & \\
\hline Abdominal & 85272 (62.5) & $87786(54.2)$ & 43767 (32.8) & $41153(34.0)$ & $<0.001$ \\
\hline Cardiac & 20247 (14.8) & 38339 (23.6) & 46448 (34.8) & 42209 (34.9) & $<0.001$ \\
\hline Retroperitoneal & $7668 \quad(5.6)$ & $7703(4.8)$ & $15631(11.7)$ & $9195 \quad$ (7.6) & $<0.001$ \\
\hline Thoracic & $4376 \quad(3.2)$ & $8307(5.1)$ & $11697(8.8)$ & $13493(11.2)$ & $<0.001$ \\
\hline Vascular & 18890 (13.8) & $19978(12.3)$ & 15725 (11.8) & $14788(12.2)$ & $<0.001$ \\
\hline \multicolumn{6}{|l|}{ Age, yr } \\
\hline$<45$ & $34180(25.0)$ & $30380(18.7)$ & $18921(14.2)$ & 13796 (11.4) & $<0.001$ \\
\hline $45-64$ & 48385 (35.5) & 59770 (36.9) & 50915 (38.2) & $45896(38.0)$ & \\
\hline$\geq 65$ & 53888 (39.5) & 71963 (44.4) & $63432(47.6)$ & $61146(50.6)$ & \\
\hline Female sex & 67027 (49.1) & 65373 (40.3) & 59594 (44.7) & $50350(41.7)$ & $<0.001$ \\
\hline \multicolumn{6}{|l|}{$\begin{array}{l}\text { Charlson Comorbidity Index, } \\
\text { score } \neq\end{array}$} \\
\hline 0 & $107929(79.1)$ & $121653(75.0)$ & $98674(74.0)$ & 86075 (71.2) & $<0.005$ \\
\hline 1 & $14647(10.7)$ & $20026(12.4)$ & $16172(12.1)$ & 12560 (10.4) & \\
\hline 2 & $7483 \quad(5.5)$ & $11009(6.8)$ & $9653(7.2)$ & $11691 \quad(9.7)$ & \\
\hline$\geq 3$ & $6394 \quad(4.7)$ & $9425(5.8)$ & $8769(6.6)$ & 10512 & \\
\hline \multicolumn{6}{|l|}{ Comorbid medical conditions§ } \\
\hline Diabetes mellitus & $15152(11.1)$ & $22272(13.7)$ & $23679(17.8)$ & 25877 (21.4) & $<0.001$ \\
\hline Peripheral vascular disease & 2025 (1.5) & $2107(1.3)$ & $1309(1.0)$ & $643 \quad(0.5)$ & $<0.001$ \\
\hline COPD & $24113(17.7)$ & $29274(18.1)$ & $26081(19.6)$ & 23940 (19.8) & $<0.001$ \\
\hline Hypertension & 39468 (28.9) & $55378(34.2)$ & $54891(41.2)$ & $54146(44.8)$ & $<0.001$ \\
\hline Coronary artery disease & 44867 (32.9) & $65379(40.3)$ & $61526(46.2)$ & $53343(44.1)$ & $<0.001$ \\
\hline Cerebrovascular disease & $1654 \quad(1.2)$ & $2185(1.3)$ & $1761(1.3)$ & $1687 \quad(1.4)$ & $<0.001$ \\
\hline Chronic kidney disease & $2737 \quad(2.0)$ & $4010(2.5)$ & 5215 (3.9) & $6617 \quad(5.5)$ & $<0.001$ \\
\hline Liver dysfunction & $3321 \quad(2.4)$ & $4003(2.5)$ & $3562(2.5)$ & $3864 \quad$ (3.2) & $<0.001$ \\
\hline Cancer & $40337(29.6)$ & $51496(31.8)$ & $48561(36.4)$ & $49023(40.6)$ & $<0.001$ \\
\hline Congestive heart failure & 11749 (8.6) & $16207(10.0)$ & $15079(11.3)$ & $13825(11.4)$ & $<0.001$ \\
\hline \multicolumn{6}{|c|}{$\begin{array}{l}\text { Note: COPD = chronic obstructive pulmonary disease. } \\
\text { *Cochrane-Armitage test for trend. } \\
\text { †Reported percentage of surgeries per year. } \\
\text { ‡Scores were assigned using patient information from hospital records in the three years before surgery. Patients with no } \\
\text { admissions to hospital during this period were assigned a value of zero. } \\
\text { \$As defined by the presence of administrative diagnostic codes (see Appendix 1, available at www.cmaj.ca/lookup/suppl/doi:10 } \\
.1503 / \mathrm{cmaj} .110895 /-/ D C 1) .\end{array}$} \\
\hline
\end{tabular}


night stay at an acute care institution from Jan. 1, 1995, to Dec. 31, 2009. Similar to previous classification methods, we categorized surgical procedures according to anatomy: abdominal, cardiac, retroperitoneal, thoracic and vascular. ${ }^{23} \mathrm{We}$ excluded emergent or urgent surgeries from analysis because acute kidney injury may occur before such procedures. We defined the remaining surgeries as "elective." We excluded nephrectomy and kidney transplantation because they have different mechanisms of acute and chronic kidney disease. In addition, we excluded all surgeries for the purpose of placing dialysis access.

Some patients had multiple eligible admissions to hospital during the study period; for these patients, we selected one admission at random to avoid clustering in the analysis. If more than one surgical procedure was done during the same stay in hospital, we selected the first surgery to avoid selecting any urgent or emergent surgery done after an initial elective surgery. To facilitate the assessment of new kidney injury after surgery, we excluded patients with any evidence of dialysis or renal transplantation in the three years preceding surgery. Finally, we excluded one hospital from our analysis because patients there were routinely given a single treatment of intraoperative dialysis if they were undergoing cardiopulmonary bypass surgery.

\section{Outcomes}

Our primary outcome was acute dialysis, defined as the receipt of at least one dialysis treatment in the 14 days after surgery. Among patients who received acute dialysis, we considered the time at which dialysis started after surgery and the modality used (i.e., intermittent hemodialysis, peritoneal dialysis and all forms of continuous renal replacement therapy). Our secondary outcomes were death within 90 days after surgery and chronic dialysis among patients who had received acute dialysis and who survived beyond 90 days. We defined chronic dialysis as receipt of dialysis on at least two separate days between 90 and 120 days after surgery.

\section{Statistical analysis}

We categorized the study period into four eras: 1995-1997, 1998-2001, 2002-2005 and 20062009. We categorized age using cutoffs from the 2006 Census of Canada. ${ }^{24}$ We compared patient characteristics and type of surgery over time, on an annual basis, using the Cochrane-Armitage test for trend. We used multivariable logistic regression to examine the association between year of surgery and outcome, adjusting for the type of surgery and patient characteristics (Table 1). We used Wilson scores to determine $95 \%$ confidence intervals (CIs) for single proportions. ${ }^{25}$

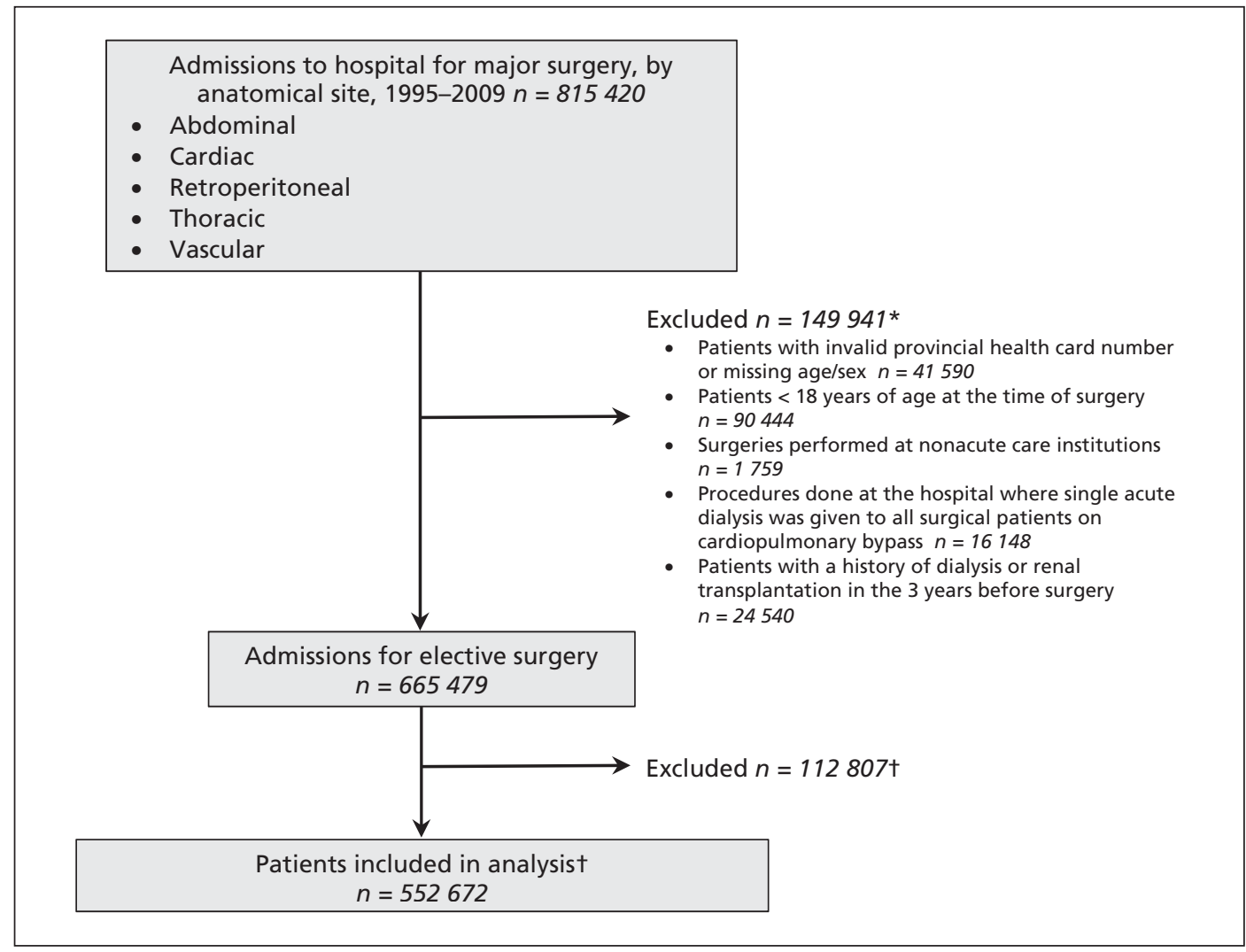

Figure 1: Selection of patients for the study. *Patients may have had more than one reason for exclusion. tFor patients who underwent multiple surgeries during the study period, one surgery was randomly selected for the analysis. 


\section{Results}

We included 552672 patients who underwent surgery at 118 hospitals (Figure 1). The age and comorbidity of patients who had elective major surgery increased during the study period (Table 1). For example, comparing the periods 1995-1997 to 2006-2009, the proportion of patients aged 65 years and older increased from
$39.5 \%$ to $50.6 \%$, as did the proportions of patients with diabetes (from $11.1 \%$ to $21.4 \%$ ), hypertension (from $28.9 \%$ to $44.8 \%$ ), coronary artery disease (from $32.9 \%$ to $44.1 \%$ ) and chronic kidney disease (from $2.0 \%$ to $5.5 \%$ ). Each of these characteristics was independently associated with postoperative acute dialysis (Figure 2). During the study period, the annual number of abdominal surgeries meeting our inclusion

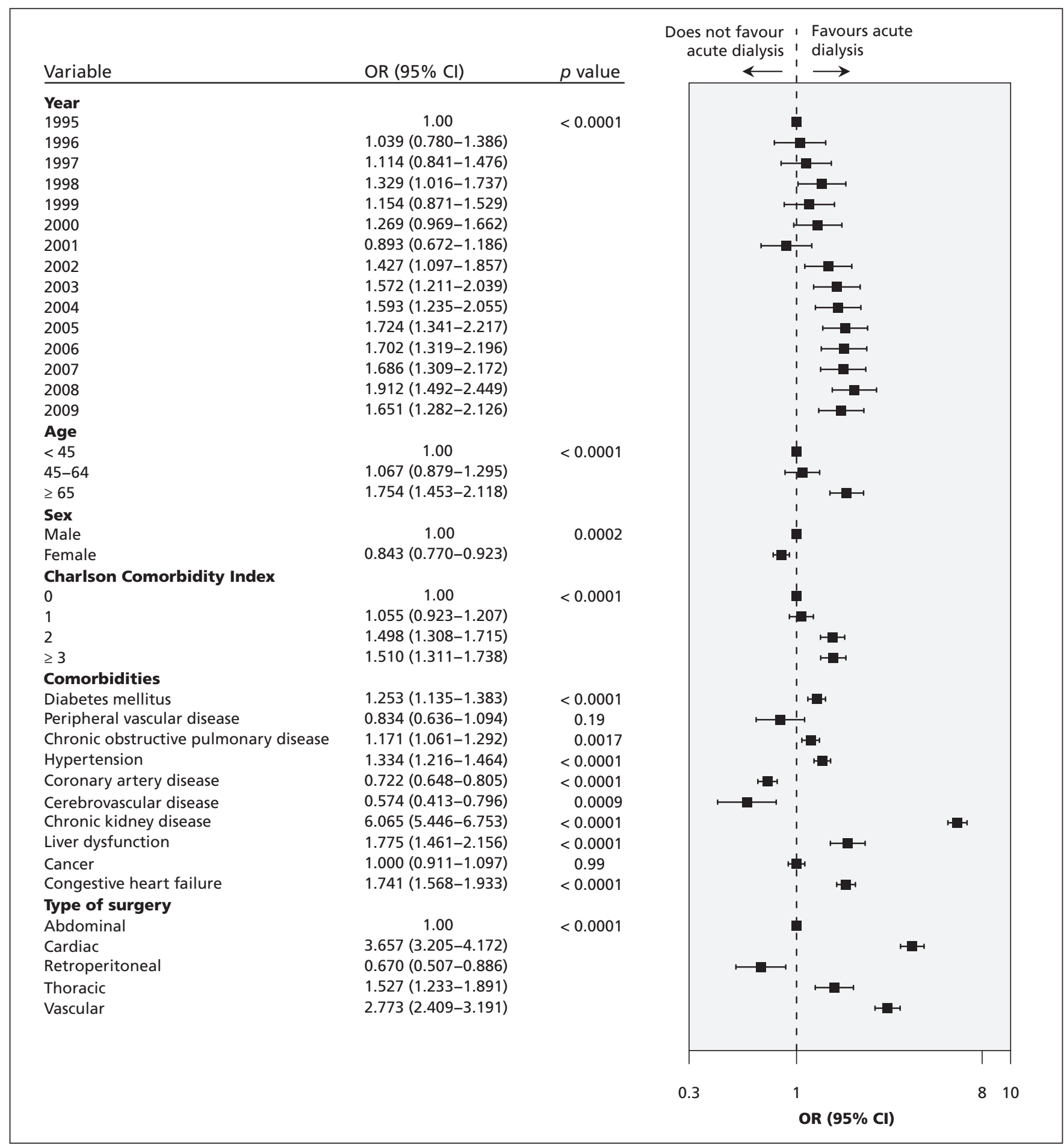

Figure 2: Multivariable logistic regression model for postoperative acute dialysis. $\mathrm{Cl}=$ confidence interval, $\mathrm{OR}=$ odds ratio. 
criteria decreased substantially, the annual number of vascular surgeries decreased slightly, and the annual numbers of cardiac, thoracic and retroperitoneal surgeries increased (Table 1).

\section{Acute dialysis}

A total of 2231 patients $(0.4 \%)$ received acute dialysis within 14 days after surgery. The incidence of acute dialysis rose steadily from $0.2 \%$ in 1995 (95\% CI $0.15-0.2)$ to $0.6 \%$ in 2009 (95\% CI 0.6-0.7). This increase was evident across all age strata (Figure 3A) and occurred primarily after cardiac and vascular surgery (Figure 3B). In recent years, as many as 1 in 80 patients had their cardiac surgery complicated by acute dialysis, compared with 1 in 390 in 1995. Similarly, in recent years, as many as 1 in 85 patients had their vascular surgery complicated by acute dialysis, compared with 1 in 230 in 1995. The rising annual trend in acute dialysis was prominent in unadjusted analysis and remained significant after statistical adjustment for patient and surgical characteristics (Figure 3C; compared with 1995, adjusted odds ratio [OR] in 2009 was $1.7,95 \%$ CI $1.3-2.1$ ). The start of acute dialysis occurred earlier after surgery in recent years. In 1995, the first treatment was done a median of 5 (interquartile range [IQR] 3-9) days after surgery, compared with a median of 2 (IQR 1-6) days after surgery in 2009 (Figure 4).

The types of dialysis used changed over time (Figure 5). Among all patients who received dialysis, $79.9 \%$ received at least one postoperative treatment using intermittent hemodialysis (data not shown). In $1995,37.6 \%$ of patients received at least one treatment using peritoneal dialysis, a modality rarely used for acute dialysis after 1998. The use of continuous renal replacement therapy increased during the study period. Among patients receiving acute dialysis, 9.7\% received at least one treatment with continuous renal replacement therapy in 1995, compared with $45.1 \%$ of patients in 2009 .

\section{Death and chronic dialysis}

Of the 2231 patients who received acute dialysis, 937 died within 90 days after surgery $(42.0 \%$, 95\% CI 40.0-44.1). There was no appreciable change in 90-day mortality over time (Appendix 3 , available at www.cmaj.ca/lookup/suppl/doi :10.1503/cmaj.110895/-/DC1) (Figures 6A and $6 \mathrm{~B}, p=0.6)$. Of the 1294 patients who received acute dialysis and survived more than 90 days, 352 subsequently required chronic dialysis (27.2\%, 95\% CI 24.8-29.7). There was no appreciable change in the incidence of chronic dialysis over time (Figures 7A and 7B, $p=0.5$ ).

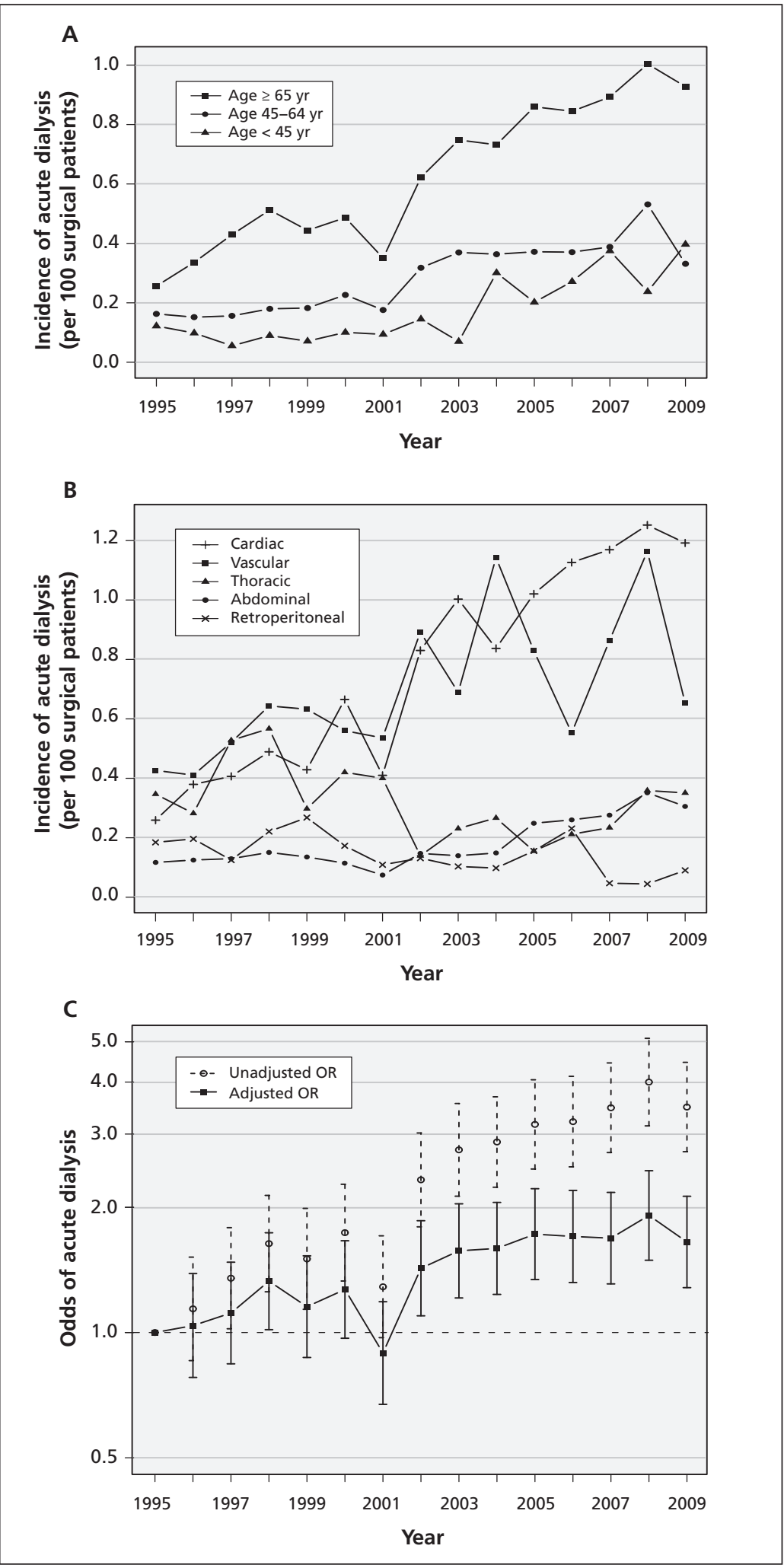

Figure 3: Incidence of postoperative acute dialysis, 1995-2009, (A) by age and (B) by type of surgery. (C) Association between year of surgery and acute dialysis after surgery, using 1995 as the reference year. Error bars indicate $95 \%$ confidence intervals. The model was adjusted for year, age, Charlson Comorbidity Index, comorbid medical conditions (diabetes mellitus, peripheral vascular disease, chronic obstructive pulmonary disease, hypertension, coronary artery disease, cerebrovascular disease, chronic kidney disease, liver dysfunction, cancer, congestive heart failure) and type of surgery. $\mathrm{OR}=$ odds ratio. 


\section{Interpretation}

Older patients and people with multiple comorbidities are increasingly undergoing elective major surgery. In parallel to the increasing presence of risk factors for acute kidney injury among surgical candidates, the incidence of

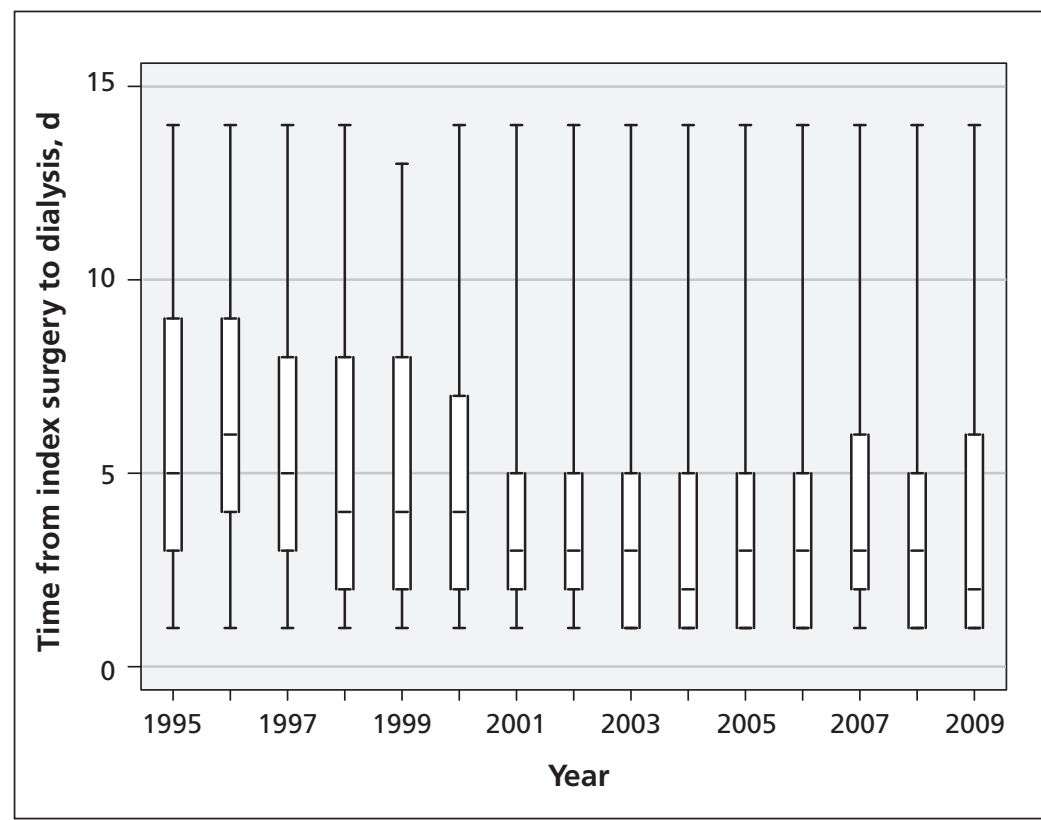

Figure 4: Time (mean, d) from index surgery to first dialysis treatment. Error bars indicate minimum and maximum values, and boxes indicate values for the interquartile range.

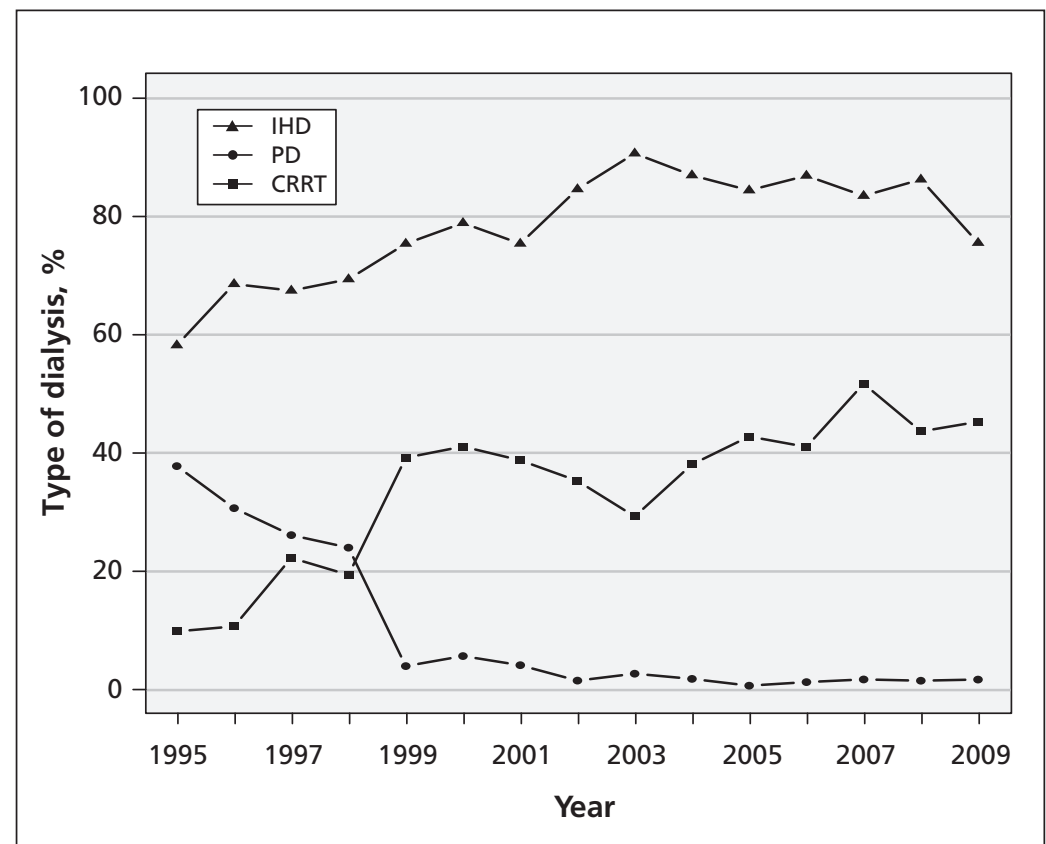

Figure 5: Incidence of acute dialysis, 1995-2009, by type of dialysis received. Type of dialysis was defined as receipt of at least one treatment using the specified modality in the 14 days postsurgery. Because a patient could receive treatment with more than one modality during the postoperative period, the percentages of all modalities in a given year may exceed $100 \%$. CRRT $=$ continuous renal replacement therapy, IHD = intermittent hemodialysis, PD = peritoneal dialysis. acute kidney injury treated with dialysis may be increasing despite general advances in surgery. In a large representative sample of five types of surgery, we saw a threefold increase in the use of acute dialysis over a period of 15 years. This increase occurred primarily among patients undergoing cardiac and vascular surgeries. Almost half the patients who received acute dialysis died within 90 days of their surgery. Among those who survived, one in four did not recover kidney function and required chronic dialysis. These latter outcomes remained consistently poor over time.

Our results may have several explanations. It is reasonable to assume that there may be a higher risk of acute kidney injury among older and increasingly comorbid patients. In addition, less invasive alternatives are developing such that only those patients with the worst prognosis may be triaged to receive surgery. For example, patients who undergo cardiac surgery today may primarily be those with diffuse coronary lesions not amenable to angioplasty, or those who need concomitant valve repair; ${ }^{26}$ such patients might be expected to have higher rates of acute kidney injury than their predecessors. Furthermore, our inclusion criteria for elective surgery required that patients stay in hospital overnight. However, compared with 10 years ago, contemporary patients who undergo less complex surgeries may no longer require an overnight stay in hospital. Accordingly, the increase in minimally invasive surgeries (e.g., laparascopic cholecystectomy) is consistent with the marked decrease in abdominal surgeries requiring an overnight stay. ${ }^{27}$

The median time to the start of dialysis decreased during the study period, from five days to two days after surgery. Patients with preoperative chronic kidney disease are among those at highest risk for acute kidney injury, and the proportion of these patients having major surgery increased with time. This group, with reduced renal reserve, may be contributing to trends in starting dialysis earlier. Alternatively, nephrologists today may be consulted earlier in the course of a patient's illness than before, dialysis may be more readily available, and the threshold for starting dialysis may be lower. ${ }^{28-31}$ This last practice may have been influenced by recent studies suggesting that starting dialysis earlier may improve prognosis. ${ }^{29,32-35}$ Unfortunately, despite the trends in increasing use of dialysis and starting it earlier, survival has remained poor. Unchanging mortality and rates of chronic dialysis also suggest these trends reflect a lower threshold for starting treatment. 


\section{Comparison with other studies}

In a study using data from the United States' Nationwide Inpatient Sample, involving 7.3 million cardiac surgeries, the incidence of acute dialysis increased from $0.2 \%$ in 1988 to $0.6 \%$ in 2003. ${ }^{11}$ Using data from about 35000 cardiac surgeries done at a single centre in Cleveland,
Ohio, Thakar and colleagues also showed an increase in the incidence of acute dialysis, from $1.5 \%$ in 1993 to $2.0 \%$ in $2002 .{ }^{10}$ Unlike our results, a decrease in mortality after acute dialysis was described in both studies, from 61\% in 1993 to $49 \%$ in $2002,{ }^{10}$ and from $47 \%$ in 1988 to $30 \%$ in 2003. ${ }^{11}$ Both of these studies described in-
A



B

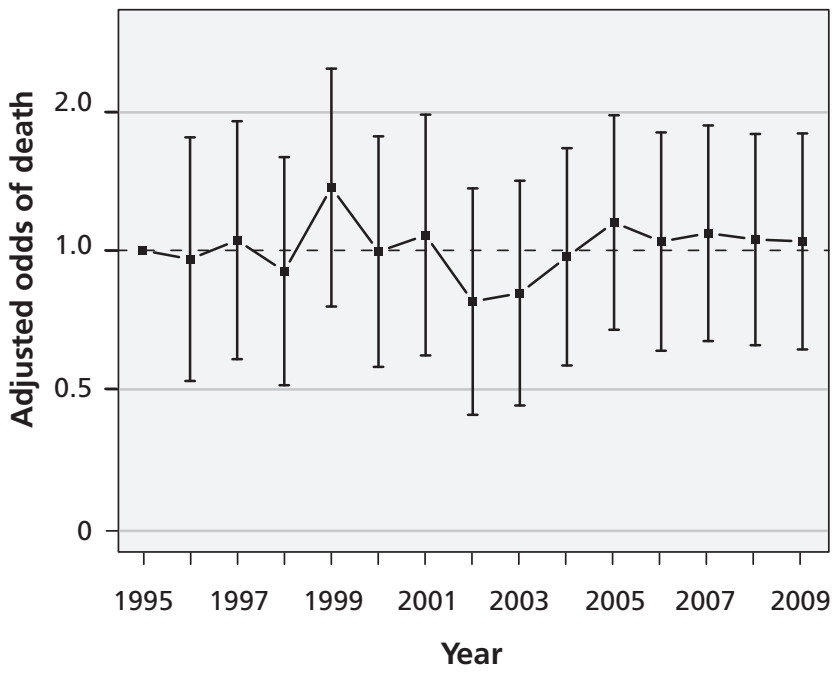

Figure 6: (A) Incidence of death at 90 days among patients who received acute dialysis after surgery. (B) Association between year of surgery and death among patients who received acute dialysis, using 1995 as the reference year. Error bars indicate $95 \%$ confidence intervals. The model was adjusted for year, age, Charlson Comorbidity Index, comorbid medical conditions (diabetes mellitus, peripheral vascular disease, chronic obstructive pulmonary disease, hypertension, coronary artery disease, cerebrovascular disease, chronic kidney disease, liver dysfunction, cancer, congestive heart failure) and type of surgery.
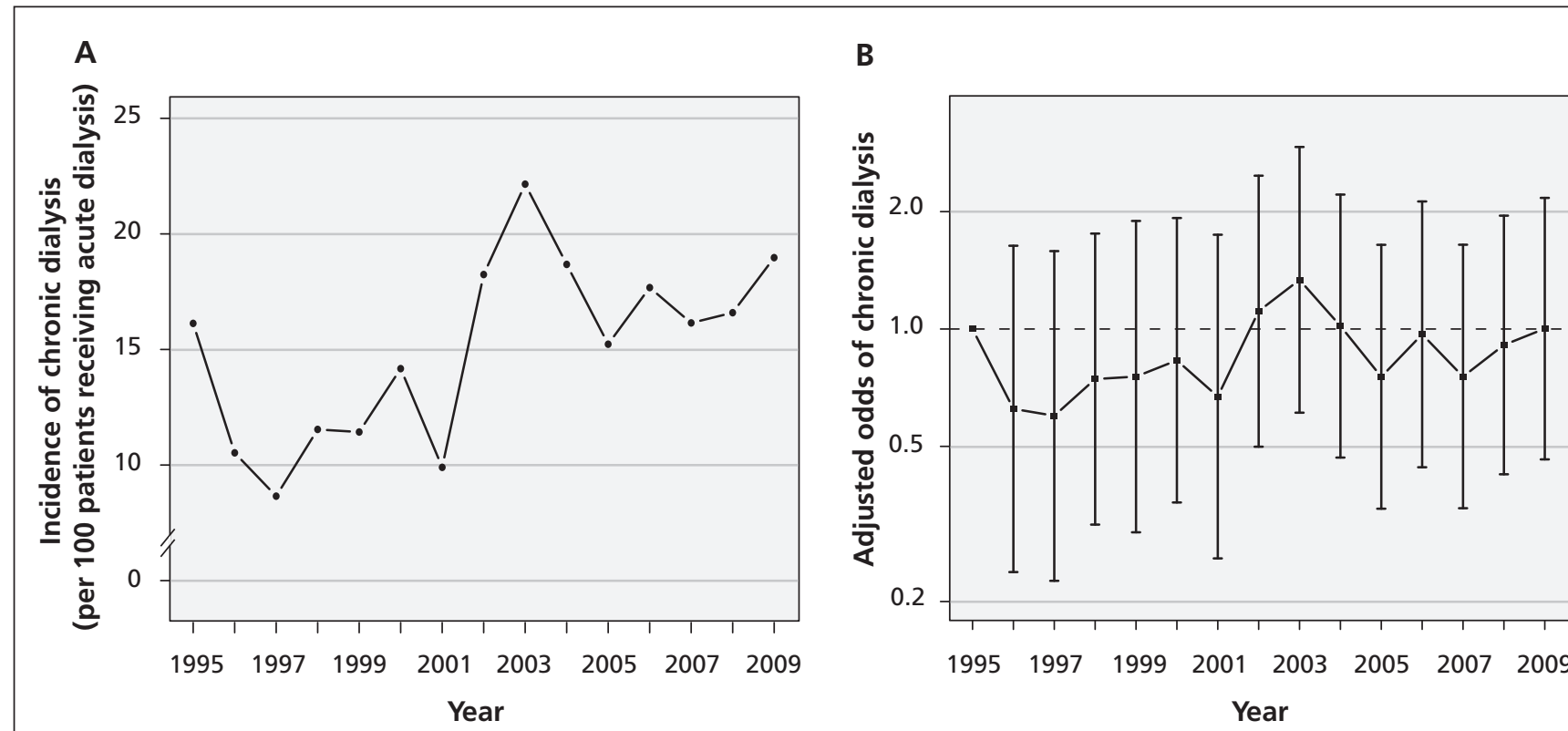

Figure 7: (A) Incidence of chronic dialysis among patients who received acute dialysis after surgery and survived beyond 90 days. (B) Association between year of surgery and chronic dialysis among patients who received acute dialysis, using 1995 as the reference year. Error bars indicate $95 \%$ confidence intervals. The model was adjusted for year, age, Charlson Comorbidity Index, comorbid medical conditions (diabetes mellitus, peripheral vascular disease, chronic obstructive pulmonary disease, hypertension, coronary artery disease, cerebrovascular disease, chronic kidney disease, liver dysfunction, cancer, congestive heart failure) and type of surgery. 
hospital mortality rather than 90-day mortality, which is a potential reason for the different results.

Little attention has been paid to patients who fail to recover kidney function after receiving postoperative dialysis for acute kidney injury. One study from Boston, Massachusetts, described a need for chronic dialysis in $64 \%$ of 13847 patients who received acute dialysis following coronary artery bypass grafting. ${ }^{36}$ Another study from Swansea, Wales, found that only $2.2 \%$ of 98 patients who received continuous renal replacement therapy after cardiac bypass subsequently received long-term renal support. ${ }^{37}$

\section{Strengths and limitations}

We were able to study data from more than 500000 patients who underwent a variety of surgical procedures in more than 100 hospitals. In addition, procedural codes for both surgery and dialysis are well-documented, with little chance of misclassification.

We did not have data on patient characteristics at the time dialysis was started to determine whether thresholds for starting dialysis have changed. Furthermore, we did not have access to the results of laboratory investigations. Instead, we used database codes to assess preoperative chronic kidney disease, recognizing that these diagnostic codes are specific but not sensitive. Similarly, given the limitations of diagnostic codes for acute kidney injury, we did not examine the outcome of perioperative acute kidney injury that was not treated with dialysis. ${ }^{16} \mathrm{We}$ were thus unable to contrast the outcomes over time of similar patients who did and did not receive acute dialysis. Finally, secular changes in database codes and coding practices may have influenced some of our results; however, most codes were stable during our period of interest and were unlikely to affect the overall trends we saw.

\section{Conclusion}

This study shows an important increase in the complication of severe acute kidney injury treated with dialysis after cardiac and vascular surgeries. Outcomes for patients who receive acute dialysis after surgery remain poor. To date, interventional studies to prevent acute kidney injury in the surgical setting have been limited in their size, rigor and success. ${ }^{38}$ Our results should prompt renewed efforts to develop and test interventions to prevent severe acute kidney injury and to attenuate the high burden of death and end-stage renal disease after such injury has occurred.

\section{References}

1. Weiser TG, Regenbogen SE, Thompson KD, et al. An estimation of the global volume of surgery: a modelling strategy based on available data. Lancet 2008;372:139-44.

2. Chertow GM, Burdick E, Honour M, et al. Acute kidney injury, mortality, length of stay, and costs in hospitalized patients. J Am Soc Nephrol 2005;16:3365-70.

3. Waikar SS, Curhan GC, Wald R, et al. Declining mortality in patients with acute renal failure, 1988 to 2002. J Am Soc Nephrol 2006; 17:1143-50.

4. Xue JL, Daniels F, Star RA, et al. Incidence and mortality of acute renal failure in Medicare beneficiaries, 1992 to 2001. J Am Soc Nephrol 2006; 17:1135-42.

5. Hsu CY. Where is the epidemic in kidney disease? J Am Soc Nephrol 2010;21:1607-11.

6. Boyle JM, Moualla S, Arrigain S, et al. Risks and outcomes of acute kidney injury requiring dialysis after cardiac transplantation. Am J Kidney Dis 2006;48:787-96.

7. Chertow GM, Lazarus JM, Christiansen CL, et al. Preoperative renal risk stratification. Circulation 1997;95:878-84.

8. Filsoufi F, Rahmanian PB, Castillo JG, et al. Predictors and early and late outcomes of dialysis-dependent patients in contemporary cardiac surgery. J Cardiothorac Vasc Anesth 2008;22:522-9.

9. Frost L, Pedersen RS, Lund O, et al. Prognosis and risk factors in acute, dialysis-requiring renal failure after open-heart surgery. Scand J Thorac Cardiovasc Surg 1991;25:161-6.

10. Thakar CV, Worley S, Arrigain S, et al. Improved survival in acute kidney injury after cardiac surgery. Am J Kidney Dis 2007; 50:703-11.

11. Nicoara A, Patel UD, Phillips-Bute BG, et al. Mortality trends associated with acute renal failure requiring dialysis after $\mathrm{CABG}$ surgery in the United States. Blood Purif 2009;28:359-63.

12. Braams R, Vossen V, Lisman BA, et al. Outcome in patients requiring renal replacement therapy after surgery for ruptured and non-ruptured aneurysm of the abdominal aorta. Eur J Vasc Endovasc Surg 1999;18:323-7.

13. Fertmann J, Wolf $\mathrm{H}$, Kuchenhoff $\mathrm{H}$, et al. Prognostic factors in critically ill surgical patients requiring continuous renal replacement therapy. J Nephrol 2008;21:909-18.

14. Landoni G, Zangrillo A, Franco A, et al. Long-term outcome of patients who require renal replacement therapy after cardiac surgery. Eur J Anaesthesiol 2006;23:17-22.

15. Waikar SS, Wald R, Chertow GM, et al. Validity of international classification of diseases, ninth revision, clinical modification codes for acute renal failure. J Am Soc Nephrol 2006;17:1688-94.

16. Vlasschaert ME, Bejaimal SA, Hackam DG, et al. Validity of administrative database coding for kidney disease: a systematic review. Am J Kidney Dis 2011;57:29-43.

17. Abelha FJ, Botelho M, Fernandes V, et al. Outcome and quality of life of patients with acute kidney injury after major surgery. Nefrologia 2009;29:404-14.

18. Zhao YY, Weir MA, Manno M, et al. New fibrate use and acute renal outcomes in elderly adults: a population-based study. Ann Intern Med 2012;156:560-9.

19. Molnar AO, Coca SG, Devereaux PJ, et al. Statin use associates with lower incidence of acute kidney injury after major elective surgery. J Am Soc Nephrol 2011;22:939-46.

20. Lam NN, Jain AK, Hackam DG, et al. Results of a randomized controlled trial on statin use in dialysis patients had no influence on statin prescription. Kidney Int 2009;76:1172-9.

21. Garg AX, Meirambayeva A, Huang A, et al. Cardiovascular disease in kidney donors: matched cohort study. BMJ 2012;344: e1203.

22. Alter DA, Naylor CD, Austin P, et al. Effects of socioeconomic status on access to invasive cardiac procedures and on mortality after acute myocardial infarction. N Engl J Med 1999;341:1359-67.

23. Redelmeier D, Scales D, Kopp A. Beta blockers for elective surgery in elderly patients: population-based, retrospective cohort study. BMJ 2005;331:932.

24. Statistics Canada. Profile of age and sex for Canada, provinces, territories, census divisions and census subdivisions, 2006 Census. Catalogue No. 94-575-XCB2006001.

25. Wilson EB. Probable inference, the law of succession, and statistical inference. J Am Stat Assoc 1927;22:209-12.

26. Hassan A, Newman A, Ko DT, et al. Increasing rates of angioplasty versus bypass surgery in Canada, 1994-2005. Am Heart $J$ 2010;160:958-65.

27. Csikesz NG, Singla A, Murphy MM, et al. Surgeon volume metrics in laparoscopic cholecystectomy. Dig Dis Sci 2010;55: 2398-405.

28. Mehta RL, McDonald B, Gabbai F, et al. Nephrology consultation in acute renal failure: Does timing matter? Am J Med 2002; 113:456-61.

29. Shiao CC, Wu VC, Li WY, et al.; National Taiwan University 
Surgical Intensive Care Unit-Associated Renal Failure Study Group. Late initiation of renal replacement therapy is associated with worse outcomes in acute kidney injury after major abdominal surgery. Crit Care 2009;13:R171.

30. Swaminathan M, Shaw AD, Phillips-Bute BG, et al. Trends in acute renal failure associated with coronary artery bypass graft surgery in the United States. Crit Care Med 2007:35:2286-91.

31. Udani SM, Murray PT. The use of renal replacement therapy in acute decompensated heart failure. Semin Dial 2009;22:173-9.

32. Nakasuji M, Nishi S, Nakasuji K, et al. Early continuous venovenous hemodialysis in dialysis-dependent patients after cardiac surgery: safety and efficacy. J Cardiothorac Vasc Anesth 2007; 21:379-83

33. Macedo E, Mehta RL. Early vs. late start of dialysis: it's all about timing. Crit Care 2010;14:112.

34. Carl DE, Grossman C, Behnke M, et al. Effect of timing of dialysis on mortality in critically ill, septic patients with acute renal failure. Hemodial Int 2010;14:11-7.

35. Bagshaw SM, Uchino S, Bellomo R, et al. Beginning and Ending Supportive Therapy for the Kidney (BEST Kidney) investigators. Timing of renal replacement therapy and clinical outcomes in critically ill patients with severe acute kidney injury. $J$ Crit Care 2009;24:129-40.

36. Leacche M, Rawn JD, Mihaljevic T, et al. Outcomes in patients with normal serum creatinine and with artificial renal support for acute renal failure developing after coronary artery bypass grafting. Am J Cardiol 2004;93:353-6.

37. Luckraz H, Gravenor MB, George R, et al. Long and short-term outcomes in patients requiring continuous renal replacement therapy post cardiopulmonary bypass. Eur J Cardiothorac Surg 2005; 27:906-9.

38. Park M, Coca SG, Nigwekar SU, et al. Prevention and treatment of acute kidney injury in patients undergoing cardiac surgery: a systematic review. Am J Nephrol 2010;31:408-18.

Affiliations: From the Division of Nephrology (Siddiqui, Jain, Li, Thiessen Philbrook, Garg), Department of Medicine, and the Department of Epidemiology and Biostatistics (Garg), Western University, London, Ont.; the Division of Nephrology (Siddiqui, Wald) and the Department of Health Policy, Management and Evaluation (Paterson), University of Toronto, Toronto, Ont.; the Section of Nephrology (Coca, Parikh), Yale University School of Medicine, New Haven, Conn.; the Department of Clinical Epidemiology and Biostatistics (Devereaux, Walsh, Garg), the Department of Medicine (Devereaux, Walsh), the Department of Family Medicine (Paterson) and the Department of Surgery (Whitlock), McMaster University, Hamilton, Ont.; and the Institute for Clinical Evaluative Sciences (Luo, Paterson, Garg), Toronto, Ont.

Funding: Grant support was provided by the Lawson Health Research Institute. Dr. Steven Coca was supported by a career development grant (K23DK0813) from the United States National Institutes of Health. Dr. Philip Devereaux was supported by a New Investigator Award from the Canadian Institutes of Health Research (CIHR). Dr. Arsh Jain was supported by a fellowship award from the CIHR. Dr. Ron Wald and Dr. Michael Walsh were supported by Randomized Controlled Trial Mentorship Program Awards from the CIHR. Dr. Amit Garg was supported by a Clinician Scientist Award from the CIHR. The Institute for Clinical Evaluative
Sciences receives funding from the Ontario Ministry of Health and Long-Term Care. The opinions, results and conclusions reported in this paper are those of the authors and are independent from the funding sources.

Contributors: All authors contributed to study's design, the analysis and interpretation of the data, and drafting and revising the manuscript for important intellectual content. All of the authors approved the final version submitted for publication.

Acknowledgements: The authors thank Dr. Ping Li and Dr. Ann Young for their help and support. 